\title{
UNA EVALUACIÓN DE LAS ACTITUDES RELACIONADAS CON LA CIENCIA
}

\author{
VÁZQUEZ ALONSO, A. ${ }^{1}$ y MANASSERO MAS, M.A. ${ }^{2}$ \\ ${ }^{1}$ Servicio de Inspección, Ministerio de Educación. \\ ${ }^{2}$ Departamento de Psicología, Universidad Islas Baleares.
}

\begin{abstract}
SUMMARY
A taxonomy of science related attitudes with four basic dimensions (global image, social aspects, school aspects, and science characteristics) has been assessed by means of a questionnaire, made independently of the taxonomy. The aim is to show how the use of the taxonomy allows us to overcome the methodologic and conceptual difficulties in the measurement of attitudes. Applied to a representative sample of secondary and university students, the results show a moderately positive attitude and some significant attitudinal differences among the taxonomy's dimensions. The image of science dimension has the best attitudinal mean score, while the social aspect dimension has the worst score. Attitudes towards school aspects of science and the characteristics of scientific knowledge elicit intermediate attitudinal scores. Science teachers can find in this paper some patterns and instruments to assess the students' science related attitudes.
\end{abstract}

\section{INTRODUCCIÓN}

Las actitudes en la educación se pueden considerar, a la vez, como causa y como efecto, esto es, como determinantes y objetivos del aprendizaje: como causas del aprendizaje, las actitudes positivas/negativas favorecen/dificultan el aprendizaje; y, por otro lado, consideradas como efecto del aprendizaje, las actitudes puede ser un contenido más de aprendizaje $\mathrm{y}$, por tanto, ser educadas y aprendidas en la escuela. Una buena actitud hacia el estudio y el aprendizaje, en general, suele ser garantía de motivación, interés y esfuerzo en los estudiantes, y los profesores entienden y aprecian las actitudes en este sentido, como elementos que favorecen el rendimiento escolar; pero también las actitudes pueden ser planteadas como una consecuencia o efecto de la educación, es decir, como objetivos a conseguir por medio de fa educación. El profesorado suele ser consciente del papel de las actitudes como causa favorecedo- ra de los aprendizajes, pero con frecuencia se ignora o minimiza su papel como objetivos y contenidos educativos y, en consecuencia, no se planifican ni se evalúan, dejándolas fuera del currículo escolar explícito. El currículo de la reforma educativa incluye las actitudes como nuevos contenidos del currículo escolar, independientes de los conocimientos, sentando las bases para su educación en la escuela.

Desde una perspectiva de investigación, el tema de las actitudes generales (sociales y políticas) ha sido siempre muy controvertido y dinámico, especialmente por lo que se refiere a su conceptualización y medición, pero a lo largo de la última década, en el marco teórico de la psicología social, se ha logrado una cierta estabilización. Algo análogo ha sucedido con la investigación de las actitudes relacionadas con la ciencia, cuyo desarrollo 
ha sido más tardio y discurrido de espaldas a la psicología social, de modo que algunas importantes revisiones (Schibeci, 1984; Shriglcy y Koballa, 1992) coinciden al señatar los importantes defectos metodológicos observados en la evaluación de las actitudes en ciencias, atribuyéndolo a la falta đe un marco teórico apropiado. Entre los más importantes defectos metodologicos están la falta de precisión en la definición del objeto de actitud que se mide (cuando esta definición existe) y la escasa valider/fiabilidad de los instrumentos construidos. En el caso de las actitudes relacionadas con la ciencia, esta problemática afecta a la propia definición de muchos objetos de actitud relacionados con aquélla, especialmente en el caso de la metodología científica. La filosofía y epistemología de la ciencia han contribuido a clarificar sus características y su naturaleza, pero han sido variables dialécticamente a lo largo del tiempo; así, por ejemplo, el positivismo considera a la ciencia un tipo de conocimiento infalible, mientras que los análisis históricos actuales ponen cl acento en el carácter falible y cambiante. Por tanto, a la hora de evaluar la adecuación de una actitud hacia la ciencia en este punto, un positivista considerará mejor una actitud infalibilista, mientras que un relativista considerará más apropiada una actitud más crítica sobre la falibilidad de la ciencia.

La medida de las actitudes es un área aquejada de numerosos problemas conceptuales y dificultades metodológicas, que es también extensiva a la medida de las actitudes relacionadas con la ciencia, como han puesto de manifiesto algunas revisiones periódicas (Gardner, 1975; Gauld y Hukins, 1980; Schibeci, 1984; Shrigley y Koballa, 1992). El problema central es la dificultad de la medición de las actitudes (Shrigley y Koballa, 1984), que ha sido fuente de frecuentes controversias y crisis sobre la validez y fiabilidad de los instrumentos utilizados (Bratt, 1984; Munby, 1983; Zeidler, 1984). Esta problemática ha conducido al desarrollo de numerosos instrumentos de evaluación de actitudes relacionadas con la ciencia (Munby contabiliza hasta 204 instrumentos en el período 1967.77), que a su vez realimenta el problema, por la endeble 2 de muchos de ellos. Los problemas más frecuentes en la medida de actitudes son: la tendencia de los alumnos a responder para satisfacer las expectativas que creen que su profesor tiene sobre ellos (que puede ser evitado respondiendo anónimamente); los problemas semánticos de significado y comprensión de la terminología empleada (sobre todo palabras como frecuente, mucho, poco...), que deben ser eliminados con una terminología lo más precisa posible o una buena explicación a los alumnos; pero sobre todo el problema más grave es la inadecuación de criterio, es decir, la falta de ajuste entre el método o instrumentos elegido para la medida y el objeto actitudinal a medir, que es el más difícil de valorar y corregir (Gauld y Hukins, 1980).

I.a producción nacional de investigaciones sobre actitudes relacionadas con la ciencia no es ajena a esta problemática: falta de un marco de referencia teórico, las deficiencias de los instrumentos de evaluación y las dificultades en la definición del objeto de la actitud. La mayoría están centradas en la actitud hacia el estudio y aprendizaje de las ciencias, tal vez por ser el concepto más elemental, familiar y útil al profesorado, hasta el punto de que en algunos trabajos se acepta implícita y acríticamente como el único objeto de actitud (Acevedo, 1993a; Arana, Escudero, Garcés y Palacian, 1985; Espinosa y Román, 1991, 1995; Gómez y Cervera, 1989; Moreno y Gil, 1987; Ortega et al. 1992; Serrano, 1988; Soneira, Lires y Pizarro, 1993), en tanto que un número menor entra en el análisis de las actitudes más propiamente científicas (Acevedo, 1993b; Serrano, 1986) y los temas Ciencia-Técnica-Sociedad (Âlvarez, Soneira y Pizarro, 1993; Guasch, de Manuel y Gras, 1993; Solbes y Vilches, 1989, 1992).

En síntesis, los puntos débiles de la investigación de las actitudes relacionadas con la ciencia se concretan en utilizar un concepto de actitud reducido a considerar las actitudes como medio para un mejor aprendizaje de la ciencia (mientras que no se considera tanto la educación de las actitudes relacionadas con la ciencia como contenido específico de la educación en ciencias), y la falta de una definición precisa de los distintos objetos posibles de actitud relacionada con la ciencia. Ambos aspectos están muy relacionados, puesto que la falta de precisión en la definición de qué es una actitud hacia la cienciat, por defecto, en la mayoría de los casos, se reduce a la actitud hacia el aprendizaje de la ciencia en la escuela, que es el concepto de actitud más intuitivo e ingenuo. Buena actifud significa buena disposición a aprender ciencia y mala actitud se identifica con algún tipo de rechazo o renuencia ante las actividades plantcadas en la escuela para aprender ciencia. Fn otros casos donde se realiza una medida empírica de actitudes hacia la ciencia, la actitud tampoco se define explícitamente, pero cl tipo de cuestiones planteadas para su evaluación determina eI tipo de actitud que se está midiendo.

Desde el punto de vista conceptual y metodológico, es importante definir con claridad el objeto de cada actitud, como base para construir instrumentos de evaluación válidos y como paso imprescindible para saber de qué actitud se habla cuando se habla de actitudes. Así, la actitud hacia el aprendizaje de la ciencia en la escuela es un objeto de actitud diferente de la actitud hacia la ciencia como cuerpo de conocimiento e investigación de la naturaleza, de modo que un alumno puede tener una actitud muy positiva hacia la ciencia como investigación, manifestada en leer libros, artículos, ver películas y buscar información sobre temas de su interés, pero carecer de interés por aprender la ciencia que le ofrecen en la escuela. Dentro de cada una de esas dimensiones de actitudes hacia la ciencia, por ejemplo, como cuerpo de conocimiento, las actitudes pueden ser tan opuestas como las que se derivan de creer que la ciencia es una disciplina infalible, absoluta o acumulativa, o por el contrario, falible, relativa o evolutiva. Una actitud infalibilista de la ciencia implica conductas de memorización, formulismos, aprendizajes mecánicos, etc., mientras que una actitud falibilista supone una visión más crítica, susceptible de discutir las ideas, aprendizajes abiertos, ideas mejor fundamentadas, etc. cuyas consecuencias para y desde la educación son evidentes. 
Recientemente hemos revisado desde el punto de vista teórico toda esta compleja problemática conceptual e instrumental, que aquí no puede más que quedar insinuada por las limitaciones de espacio, proponiendo un concepto multidimensional de las actitudes relacionadas con la ciencia (en plural, porque es básico admitir que existen muchos y diferentes objetos de actitud), concretada en una taxonomía fundamentada de las mismas que permite una definición más precisa y delimitada de cada objeto de actitud (Vázquez y Manassero, 1995). Esta taxonomía constituye un paso metodológico necesario para una válida y correcta evaluación de las actitudes, y también para la aceptación de las distintas actitudes como contenidos independientes e importantes de la educación en ciencias. Las dimensiones básicas de la taxonomía son cuatro: enseñanza de la ciencia, imagen de la ciencia, incidencia social de la ciencia y características de la ciencia. A su vez, la enseñanza de la ciencia está subdividida en la ciencia escolar y resultados de la enseñanza. Y el apartado de las características de la ciencia contiene las subdimensiones de valores de la ciencia, la ciencia como empresa colectiva y la naturaleza epistemológica de la ciencia. En la filosofía básica de la concepción de esta taxonomía encontramos la consideración de la ciencia y la tecnología como disciplinas interactuantes y parcialmente superpuestas en la sociedad actual, la extraordinaria importancia de la dimensión actitudinal en los denominados temas CTS (Ciencia-Tecnología-Sociedad) y en los temas que se refieren a la comprensión de las características propias de la ciencia y la tecnología (métodos, valores, sociología, etc.).

En este artículo se ofrece una evaluación empírica de las actitudes relacionadas con la ciencia mediante un instrumento estandarizado, y que se utiliza para operacionalizar las dimensiones de la taxonomía propuesta sobre una escala de actitudes concreta, desarrollada en base a diferentes escalas anteriores. El hecho de que este instrumento haya sido construido independientemente de las dimensiones de la taxonomía para evaluar las actitudes rclacionadas con la ciencia revaioriza la potencial universalidad de la taxonomía para ser utilizada como medio de análisis y definición de las actitudes relacionadas con la ciencia, al ser adaptable a diferentes instrumentos que pueđan construirse para evaluarlas. Por tanto, la taxonomía podría servir como referencia universal para enmarcar la validez del constructo de cualquier instrumento de evaluación de actitudes relacionadas con la ciencia.

Por otro lado, este estudio tiene también el objetivo de realizar una evaluación diagnóstica de las actitudes relacionadas con la ciencia en estudiantes de todos los niveles educativos, superando la visión de las actitudes, que se reduce a considerar principalmente la actitud hacia el aprendizaje de la ciencia en ia escuela En este estudio se evalúan diferencialmente, también, las actitudes hacia las diferentes dimensiones de la taxonomía: la imagen de la ciencia, Ios temas de ciencia con incidencia social, los valores científicos, la ciencia como empresa colectiva y hacia la naturaleza del conocimiento científico.
Estos dos objetivos se podrían sintetizar en una hipótesis de trabajo que este estudio trata de verificar: EI análisis de los resultados empíricos mediante las dimensiones de la taxonomía resulta relevante y valioso, cstableciéndose diferencias entre los distintos objetos de actitud que definen cada una de las dimensiones.

\section{MÉTODO}

\section{Muestra}

La muestra de aplicación del cuestionario está compuesta por estudiantes de todos los niveles y modalidades del sistema educativo, desde la educación obligatoria hasta cursos universitarios de posgrado, existentes en cl curso 1993-94 en la isla de Mallorca. No obstante, algunos criterios previos limitaron la población representada por la muestra y, en consecuencia, condicionaron la selección muestral realizada. En primer lugar, se consideraba necesario que los alumnos tuvieran una formación y capacidad adecuadas para comprender los temas planteados en el cuestionario y por ello el límite inferior de la muestra se situó en los alumnos del último curso de la EGB, 13-14 años. Por otro lado, la progresiva implantación de los nuevos estudios derivados de la LOGSE en los centros de enseñanzas medias (bachillerato y formación profesional) en el momento de realizar la investigación dificultó el proceso de afijación proporcional para los cambiantes grupos de este nivel (incremento de grupos LOGSE y extinción de reforma y BUP), al carecer de datos poblacionales estables que permitiesen una proyección segura. La muestra obtenida es representativa de las distintas modalidades de enseñanzas medias: reforma, educación secundaria obligatoria (ESO), formación profesional (FP) y bachillerato (BUP y bachilleratos LOGSE), tanto de centros públicos como privados. Pero la afijación es sólo aproximadamente proporcional. La muestra de estudiantes universitarios se ha obtenido seleccionando aleatoriamente algunas carreras, pero con dos criterios directores: a) que hubiera alumnos đe especialidades científicas y no científicas equilibradamente $y, b$ ) para acentuar los contrastes de las actitudes estudiadas: de los cursos primero y último de las licenciaturas.

En todos los casos, para facilitar la aplicación de los cuestionarios, los participantes se obtuvieron mediante selección al azar de grupos-clase en un muestreo por cuotas entre todos los niveles existentes en el sistema educativo en ese momento. La muestra total es de 2.507 estudiantes, que, para un nivel de confianza de $95,5 \%$ y en la condición más desfavorable $(\mathrm{p}=\mathrm{q}=50 \%)$, corresponde a un margen de error muestral, respecto a la población que representa, de $+/-1,6 \%$.

\section{Instrumento}

Las actitudes relacionadas con la ciencia son medidas en este estudio con una versión propia en casteliano del 
Wareing Attitudes toward Science Protocol, una escala tipo I.ikert desarrollada por Wareing (1982), que tiene en cuenta diversos instrumentos anteriores para evaluar actitudes, tales como el Test of Understanding Science de Klopfer y Cooley (1961), la escala Attitudes toward Science and Scientific Careers de Alien (1959), el test proycctivo Projective Test of Attitudes construido por Lowery (1966) y la escala Science Support Scale de Schwirian (1968). Se intenta superar los defectos debidos a éstos, tales como la excesiva generalización en la categorización y definición del objeto de las actitudes, la falta de diferenciación entre los componentes afectivo y cognitivo y la ausencia de una adecuada adaptación a la realidad de las aulas escolares. Asimismo se han tenido en cuenta otros instrumentos posteriores tales como las escalas Scientific Attitude Scale (SAS) desarrollados y mejorados por Murphy (1968) y Billeh y Zakariades (1975), el Weinhold's Attitude Scale (Weinhold, 1970) y el Test On Scientific Attitude (TOSA) de Kozlow y Nay (1976), que remedian algunos de los defectos genéricos de la medición de actitudes y otros observados en las escalas precursoras anteriores, pero que en general han mostrado una fiabilidad baja y excesivamente variable, según las muestras de aplicación. El procedimiento de construcción partio de un banco inicial de 400 ítems, de donde se eliminaron la mitad mediante criterios de longitud y buena legibilidad, sometiendo el resto al juicio de un panel de 19 expertos (científicos y profesores de ciencias), gue clasificaron los ítems como: favorables, desfavorables o neutros respecto a la ciencia. Los ítems neutros y los que no alcanzaron un mínimo de $80 \%$ de acuerdo fueron eliminados, dejando un borrador de 79 ítems (41 favorables y 38 desfavorables), que se aplicaron a una muestra piloto de 204 alumnos del grado 4 al 12 (Wareing, 1982). l wos datos del cstudio piloto fucron cmpleados para seleccionar los 25 ítems positivos y los 25 ítems negativos, que mostraron una diferencia mayor en su puntuación media entre el tercio de alumnos de puntuaciones mayores y menores, los cuales formaton el protocolo final de 50 ítems, ordenados al azar.

I a comprobación del nivel de comprensión lectora demuestra la adecuación del protocolo para el grado 8 (equivalente en el sistema coucativo español al curso $8^{\circ}$ de $\mathrm{EGB}$ o $2^{\circ}$ curso del primer ciclo de $\mathrm{ESO}$ ), dependiendo su aplicación en grados inferiores del nivel de lectura de cada clase. La aplicacion a 806 estudiantes desde el grado 4 al 12 (Wareing, 1982) obtuvo valores de la fiabilidad según distintos métodos (Spearman-Brown, Guttman y Flanagan) muy favorables y comprendidos entre, 94 y, 84 .

Fl Protocolo de Actitudes relacionadas con la Ciencia (PAC) aplicado en este estudio está formado por un conjunto de 50 frases sencillas y cortas, a través de las cuales se mide la actitud de los estudiantes, mediante la manifiestación de su grado de acuerdo/desacuerdo con Ia frase, sobre una escala de cinco puntos tipo Likert, graduada desde «totalmente de acuerdo» hasta «totalmente en desacuerdo», con el punto central de la escala correspondiente a la opción de «indecisión» (ver texto del PAC en el apéndice).

\section{Procedimiento}

El cuestionario fuc aplicado a los estudiantes de nucstra muestra en sus grupos naturales de clase por un profesor, en la mayoría de los casos; aunque en otros casos el administrador fue un miembro del equipo de investigit ción, entrenado para ello.

Teniendo en cuenta la taxonomía de las actitudes relacionadas con la ciencia desarrollada con más detalle y fundamentación en otro lugar (Vázquez y Manassero, 1995), se han definido algunas variables diferenciadas, que reúnen las puntuaciones de varios ítems afines, y cuyo análisis será más significativo para evaluar la actitud de los estudiantes. Un aspecto importante de la taxonomía es que ciencia y tecnología (C y T) se consideran desde una perspectiva integradora, como si forma* ran un único ente (tecnociencia), que subsume ambas y hace difícil discernirlas; de hecho, la gran interacción entre ciencia y tecnología en cl mundo actual hace difícil distinguir entre ambas $y$, de hecho, muchas personas identifican la ciencia con realizaciones puramente tecnológicas, y viceversa.

I as variables definidas se corresponden con las dimensiones de la taxonomía citada, que son las siguientes: actitudes relacionadas con la enseñanza/aprendizaje de la C y T (Enseñanza), que comprende, clementos escolares de la C y $T$ (Ciencia Escolar) y los productos del aprendizaje de la C y T (Restultados Fnscũanza); actitudes relacionadas con las interacciones entre la sociedad y la $\mathrm{C}$ y $\mathrm{T}$, que comprende la imagen social de la $\mathrm{C}$ y $\mathbf{T}$ (Imagen) y temas específicos de $\mathrm{C}$ y $\mathrm{T}$ con incidencia social (Sociales); actitudes relacionadas con la naturalcza del conocimiento cientifico y técnico (Características), que comprende los valores de la ciencia (Curiosidad) y de los científicos, la naturaleza colectiva de la empresa científica (Colectiva) y las actitudes relacionadas con los métodos y procedimientos científicos (Naturaleza).

Además, existen algunos ítems del cuestionario qua inciden, con pequeños matices diferenciales, sobre un mismo tema, distinto del de las anteriores dimensiones: con ellos se han definido también algunas variables secundarias de actitudes denominadas Mundo mejor (capacidad de la ciencia para mejorar la calidad de vida general), Utilidad (beneficios de la ciencia en la vida ordinaria), Salud (papel je la ciencia en mejorar la salud y combatir enfermedades), Aburrida (grado en que Ios temas de ciencia resultan aburridos) y Planetas (estudio del sistema solar), que evalúan la actitud hacia extas cuestiones puntuales.

Las respuestas dadas a cada uno de los 50 items que componen el PAC están evaluadas en una escala tipo Likert con cinco puntos que reflejan una gradación que va desde el «acuerdo total» (5) hasta el «desacuerdo total» (1). Puesto que el cuestionario tiene ítems que están formulados positivamente y otros que están formulados negativamente, las escalas de los ítems negativos se han puntuado numéricamente en sentido inverso, de manera que las puntuaciones resultantes de cada ítem 
Tabla I

Resultados medios globales de las respuestas a los ítems del cuestionario PAC (N $=2.507$ casos).

\begin{tabular}{|c|c|c|c|c|}
\hline ITEM & CONTENIDO & MEDIA & DEV STD & DIMENSION/SLB \\
\hline Al & Mundo mejor & 3,8688 &, 8955 & SOCIAL \\
\hline $\mathrm{A} 2$ & No gusta a nadie & 4,4164 & .7038 & IMAGEN \\
\hline A3 & Ahorra tiempo y esfuerzo & 3,9605 & .8553 & SOCIAI \\
\hline A4 & Difícil de aprender & 2,8165 & 1,1168 & ENLN̄/CESCOLAR \\
\hline A5 & Cura enfermedad & 4,1970 &, 8329 & SOCIAL \\
\hline A6 & Más preocupación & 2,6239 & 1,1490 & SOCIAL \\
\hline A7 & No es aburrida & 3,7036 & 1,0320 & IMAGEN \\
\hline A8 & Ayuda a la gente & 3,0116 & 1,0953 & SOCIAL \\
\hline A 9 & Sensata & 3,4029 & .9316 & CARACTHR/NATER \\
\hline A10 & Siento mal & 4,1053 &, 9323 & IMAGEN \\
\hline AlI & Curiosidad esencial & 3,9278 &, 9948 & CARACTER/CIKIO \\
\hline $\mathrm{A} 12$ & Más salud & 3,4846 & 1,0365 & SOCIAI \\
\hline $\mathrm{A} 13$ & No resuelve encrgéticos & 3,6043 & .9244 & SOCIAL \\
\hline $\mathrm{A} 14$ & Mucha inteligencia & 2,9984 & 1,2414 & ENSEÑ/RHSUITAD \\
\hline$A 15$ & Estudian obligados & $3,475 !$ & 1,2179 & ENSEÑ/CESCOLAR \\
\hline Al6 & Conocer el mundo & 3,7491 & 1,0149 & CARACTER/NATLR \\
\hline $\mathrm{A} 17$ & Estimula la curiosidad & 3,9900 &, 8315 & CARACTER/CURIO \\
\hline$A \pm 8$ & Lo mejor trabajar & 2,4057 & 1,0041 & IMAGEN \\
\hline A19 & Valiosa & 4,0834 & 7756 & IMAGEN \\
\hline $\mathrm{A} 20$ & Conocer luna & 3,8237 &, 9501 & SOCIAL \\
\hline$A 21$ & Clases inactivas & 3,6458 & 1,0398 & ENSEÑ/CESCOI.AR \\
\hline $\mathrm{A} 22$ & Peor materia & 4,0670 &, 9046 & ENSEN/CESCOI AR \\
\hline$\wedge 23$ & Eliminar de escuela & 4,4543 &, 7642 & ENSEÑ/CESCOLAR \\
\hline$\wedge 24$ & Vida más larga & 3,5090 & 1,1195 & SOCIAL \\
\hline$\Lambda 2 S$ & Alummas robols & 4,0315 &, 9464 & ENSEÑ/RESULTAD \\
\hline A26 & Desanima la curiosidad & 4,1815 & .7881 & CARACTIR/CURIO \\
\hline$\wedge 27$ & Ayuda a pensar & 3,6302 & 9041 & CARACTER/NATUR \\
\hline A28 & Pesadilla estudio & 3,8265 & 1,0749 & ENSEN̄/CESCOLAR \\
\hline A29 & Mejor sin ciencia & 4,1185 & .8503 & ENSEÑ/RESLITAD \\
\hline $\mathrm{A} 30$ & Sin sentido no-científicos & 3,6522 & 1,0477 & ENSEÑ/RESULTAD \\
\hline $\mathrm{A} 31$ & Evita catástrofes & 3,7068 & .9931 & SOCIAL \\
\hline$\wedge 32$ & Futuro mejor & 3,6478 & 1,0154 & SOCIAI \\
\hline A33 & Prepara el futuro & 3.6534 & .8964 & SOCIAL \\
\hline A 34 & Riesgo salud & 3,6741 &, 9759 & SOClAL \\
\hline A35 & Vida menos monb́tona & 2,9593 & 1,0513 & SOCIAI \\
\hline A36 & No ir a la Luna & 4,3083 & .8681 & SOCIAL \\
\hline A 37 & Complctamente aburrida & 4,0706 &, 9464 & IMAGEN \\
\hline A 38 & Excusa juguetear & 4,1855 & .8141 & IMAGFN \\
\hline A39 & Detestable & 4,2377 &, 8795 & IMAGEN \\
\hline $\mathrm{A} 40$ & Exiremadamente útil & 3,8767 &, 9325 & ENSEN/RESUITAD \\
\hline A4l & Necesaria a todos & 3,7834 & ,9026 & SOCIAL \\
\hline A42 & Estudio curiosidad & 3,7236 &, 8632 & CARACTER/CURIO \\
\hline A43 & No utilidad & 4,4196 &, 7492 & ENSEN̄/RESULTAD \\
\hline A44 & Aceptar opiniones & 3,6617 &, 9248 & CARACTER/COLEC \\
\hline A.45 & Defiende la superstición & 3,3997 & 1,0991 & CARACTER/NATUR \\
\hline A46 & Aprecia ideas nuevas & 4,0463 & .7465 & CARACTER/COLEC \\
\hline$A 47$ & Conocimiento incambiable & 3,8955 &, 9611 & CARACTER/NATUR \\
\hline A48 & Es supersticion & 4,2457 & .8631 & CARACTERNATIR \\
\hline $\mathrm{A} 49$ & Muy interesante & 4,0511 & .8044 & IMAGEN \\
\hline A50 & Estudiar sirve & 4,0550 & .8509 & ENSEÑ/RESULTAD \\
\hline
\end{tabular}


individual y de las variables construidas, numéricamente mayores, indican una actitud más favorable hacia la ciencia y una comprensión más adecuada de la naturaleza de Ia ciencia. Para facilitar la comprensión y significación de las puntuaciones, los rangos de todas las variables de actitudes se han reducido a la misma escala básica de los ítems individuales ( 1 a 5 puntos); además, esto permite comparaciones directas entre las puntuaciones de todas las variables empleadas.

\section{RESULTADOS}

I.a tabla I contiene las puntuaciones medias y las desviaciones típicas de cada uno de los ítems que forman el cuestionario PAC obtenidos para los casos válidos de la muestra total. Se indica brevemente el contenido y el carácter inverso (-) de la formulación de los ítems del cuestionario. Como se puede observar, la mayoría de los ítems tiene medias comprendidas entre las puntuaciones 3 y 4, resultado que se puede interpretar globalmente como una tendencia de la actitud acorde con la ciencia, $y$, por tanto, moderadamente favorable a ta misma.

Los f́tems con un grado más alto de acuerdo y favorabilidad hacia la ciencia (puntuaciones superiores a 4 puntos), que indicarían una actitud muy favorable hacia la ciencia, son, paradójicamente, muchos de los f́tems cuya redacción está formulada en negativo: la ciencia no gusta a nadie, la gente se siente mal con la ciencia, la ciencia es la peor materia escolar, la ciencia debería eliminarse de las escuelas, Ios alumnos en clase de ciencias son como robots, la ciencia desanima la curiosidad, sin ciencia habría mejores estudiantes, no se debería haber enviado gente a la Luna, la ciencia es completamente aburrida, detestable, una mera excusa para juguetear, sin utilidad y es como una supersticion. Las puntuaciones medias obtenidas en ellos para Ia muestra total evidencian que los estudiantes sostienen un desacuerdo alto con estas afirmaciones y, por tanto, una actitud muy favorable hacia los aspectos de Ia ciencia reflejados en ellos.

Fntre los ítems formulados en positivo, las puntuaciones favorables más altas corresponden a los siguientes: la capacidad de la ciencia para curar las enfermedades, la ciencia es considerada algo valioso, muy interesante, que aprecia las ideas nuevas y que sirve a la gente, incluso fuera de la escuela.

De todos ellos, los fitems individuales con puntuaciones más elevadas, que indicarían la más intensa actitud favorable hacia la ciencia, son los números 3 (A nadie le gusta la ciencia), 23 (La ciencia debería ser eliminada de las escuelas) y 43 (La ciencia no tiene utilidad). Por tanto, los estudiantes se muestran en casi absoluto desacuerdo con las anteriores afirmaciones negativas sobre Ia ciencia y, complementariamente, se puede suponer que la ciencia es considerada muy útil, que gusta y que no debe ser eliminada de las escuelas, con mayor intensidad que ninguna otra cosa en relación con la ciencia.
Los ftems que reflejan una actitud menos favorable hacia la ciencia, con puntuaciones sensiblemente inferiores al punto medio de la escala (3), que representa Indecisión) son muy pocos. Uno de ellos está formulado en positivo (item 18, No hay nada mejor que trabajar en ciencia), y dos son negativos como el 4 (1 a ciencia es difícil de aprender) y el 6 (Más conocimiento científico supone más preocupaciones), alcanzando las puntuaciones más bajas los items 18 y 6 . Por tanto, parece que las actitudes más desfavorables hacia la ciencia se relacionan con los problemas y preocupaciones que genera la ciencia y Ia falta de atractivo de la ciencia como actividad o trabajo habitual.

En suma, de este primer análisis global de las puntuaciones de cada ítem se deduce una actitud global de los estudiantes moderadamente favorable y positiva hacia la ciencia, y se deduce también que esta actitud se muestra especialmente más clara y favorable hacia la ciencia en los ítems que predican alguna característica negativa o un rechazo de aquélla.

Un defecto metodológico muy frecuente en la investigación de actifudes ha sido la pretensión de medir una actitud en base al resultado de la respuesta a un único f́tem. Las consideraciones anteriores sobre los resultados de f́tems individuales no tienen esta pretension, obviamente; solamente pretenden una aproximación inicial a los resultados que sirva para ofrecer una pauta sobre las actitudes relacionadas con la ciencia de los estudiantes. Para descubrir patrones más sólidos se han definido las variables globales de conjunto que reúnen las puntuaciones de varios ítems afines, y cuyo análisis será más significativo para evaluar la actitud de los estudiantes.

La tabla 2 contiene las puntuaciones medias de las variables de conjunto definidas para el cuestionario PAC, valoradas en la misma escala empleada para medir cađa ítem del cuestionario, desde el valor 1, Desacuerdo total hasta 5, Acuerdo total. La puntuación media de la variable Actitud Total, que mide la actitud global para toda la muestra, es de 3,7754; es decir, una puntuación contenida en la parte que podríamos denominar de actitud positiva, entre el punto 3 (Indecisión) y 4 (Acuerdo), pero más próximo a la zona de acuerdo con una evaluación favorable de la ciencia, y que, en síntesis, se podría describir como una actitud moderadamente favorable hacia la ciencia.

Comparando las puntuaciones de las cuatro subescalas principales en las que se ha subdividido el cuestionario PAC (Imagen, Sociales, Enseñanza y Caracteristicas) se observa la existencia de diferencias entre ellas; los aspectos de imagen de la ciencia son los que concitan la actitud más favorable, mientras que los aspectos denominados sociales son los que tienen la actitud menos favorable; las otras dos variables, Enseñanza y Características de la Ciencia, tienen puntuaciones que corresponden a una actitud intermedia entre las anteriores. Así pues, la comparación relativa entre las puntuaciones de las variables globales definidadas en el cuestionario PAC revela que las actitudes relacionadas con la ciencia 
Tabla II

Resultados medios globales de las variables de conjunto del cuestionario PAC ( $N=2.507$ casos).

\begin{tabular}{|c|c|c|c|c|c|}
\hline $\begin{array}{l}\text { VARIABLES } \\
\text { ACTITUDES }\end{array}$ & Media & $\begin{array}{l}\text { Desv. } \\
\text { Estan. }\end{array}$ & $\begin{array}{c}\text { Número } \\
\text { ítems }\end{array}$ & Alfa & $\begin{array}{c}\text { Alfa } \\
\text { corregida }\end{array}$ \\
\hline ACTITUD TOTAL & 3,7754 & .3859 & 50 &, 8984 & .8984 \\
\hline IMAGEN & 3,9244 & .5341 & 9 &, 7867 &, 9535 \\
\hline SOCIALES & 3,6222 & .4511 & 16 & .7567 & .9453 \\
\hline YNSENAANZA & 3,8085 & .4919 & 13 & .7501 & .9203 \\
\hline CIENCIA ESCOLAR & 3,7187 & .6659 & 6 & .7239 &, 9562 \\
\hline RESULTADOS ENSZA & 3,8856 & 4680 & 7 &, 4932 & .8742 \\
\hline CARACTER,CIENCIA & 3,8321 & .4445 & 12 & .7164 &, 9132 \\
\hline CURIOSIDAD & 3,9638 &, 5837 & 4 &, 6074 & .9508 \\
\hline COLECTIVA & 3,8708 & .6452 & 2 &, 3918 &, 9415 \\
\hline NATURALE7.A & 3,7315 & .5082 & 6 & .5023 &, 8937 \\
\hline \multicolumn{6}{|c|}{ VARIABLES SECUNDARIAS } \\
\hline MUNDO MEJOR & 3,7331 & 6940 & 3 &, 6083 & .9628 \\
\hline UTILIDAD & 4,0105 & .5026 & 5 &, 5390 & .9212 \\
\hline SALUD & 3.7409 &, 7042 & 3 & ,5044 & .9443 \\
\hline ABURRIDA & 3,8953 & .8462 & 2 & ,6598 &, 9798 \\
\hline PLANETAS & 4,0714 & .7119 & 2 &, 3885 &, 9408 \\
\hline
\end{tabular}

más favorables son la que se refieren a la imagen de la ciencia y las más desfavorables son las actitudes relacio. nadas con los aspectos sociales de la ciencia.

En el caso de las subescalas de Enseñanza y Características de la ciencia, a su vez, se han definido otras escalas parciales. La subescala Enseñanza tiene definidas dos escalas parciales, la primera referida a la actitud hacia la ciencia escolar, es decir, la ciencia tal como se enseña en la escuela, y la segunda referida a la actitud hacia los resultados o productos que se obtiene de esa enseñanza. Como se deduce de sus puntuaciones medias, las actitudes hacia la Ciencia Escolar son más bajas que las actitudes hacia los Resultados de la Enseñanza de la ciencia en la escuela. En cierto modo estas puntuaciones son paradojicas, ya que se valora menos la ciencia tal como se ofrece en la escuela que los resultados que se obtienen con ella. Muy a menudo se insiste en la actitud negativa de los alumnos hacia la ciencia escolar, su aprendizaje, la metodología de enseñanza, el tipo de tareas y actividades que se realizan, las dificultades de aprendizaje, etc. Los resultados del cuestionario PAC confirman parcialmente esta tesis, ya que, de las dos escalas parciales relacionadas con la enseñanza de la ciencia, la que se refiere a la ciencia escolar tiene una puntuación relativamente más baja, aunque no resultan las más desfavorables en sentido absoluto, porque, como se ha visto, las actitudes relacionadas con los aspectos sociales de la ciencia tienen una puntuación ligeramente más baja.

La subescala Características de la ciencia tiene, a su vez, definidas tres subescaias que se refieren a: la ciencia como actividad que valora y estimula la curiosidad (Curiosidad), el conocimiento del entorno natural, el carácter de empresa colectiva de la ciencia (Colectiva) y la ontología epistemológica del conocimiento científico (Naturaleza de la ciencia). Las actitudes relacionadas con la curiosidad, como un valor de la ciencia, son las más favorables y las relacionadas con lo que es la naturaleza de la ciencia, las más desfavorables (siempre en términos relativos, entre ellas). Una de las variables que revela una actitud más favorable destaca uno de los valores típicos de la ciencia, la Curiosidad. Desde el punto de vista psicológico, la curiosidad es uno de los motores de la motivación y, por tanto, la imagen de la ciencia como algo que cultiva la curiosidad satisfecha por el conocimiento y que se basa en la curiosidad hacia la naturaleza como un elemento que mueve el desarrollo de la investigación y el conocimiento científico puede no 
sólo ser un valor científico sino también un valor educativo y motivador en la enseñanza de la ciencia.

Entre las subescalas definidas por la afinidad temática de los ítems del cuestionario $\mathrm{PAC}$, resaltan por su valor más positivo las subescalas referidas a la actitud relacionada con la investigación y el conocimiento del espacio exterior a la Tierra (Planetas), la utilidad de la ciencia (Utilidad). Es curioso observar como en momentos donde muchos programas de investigación espacial están sufriendo recortes, que limitan su capacidad de obtener resultados espectaculares o simplemente positivos, los estudiantes valoran extraordinariamente la investigación espacial, que se convierte en objeto generador de actitudes muy favorables hacia la ciencia y la tecnología. Esta interpretación es coherente también con la alta puntuación de la variable Utilidad: la percepción de utilidad de la ciencia aproxima y confunde la ciencia y la tecnología, en lo que anteriormente se ha denominado tecnociencia y que en términos filosóficos se podría etiquetar como razón práctica, la cual actúa como un generador importante de actitudes positivas entre los estudiantes.

I as diferencias entre las puntuaciones de las distintas variables del cuestionario de actitudes, aunque pequeñas en términos numéricos, debido al reducido rango de puntuaciones utilizado (entre 1 y 5 puntos), son casi todas ellas significativas cuando se comparan entre sí (empleando el estadístico $t$ para muestras apareadas). Lejos de ser un resultado extraño o paradófico debe tenerse en cuenta que la significación estadística de las diferencias entre medias determinadas por $t$, no sólo depende de la magnitud de la diferencia entre las medias de las variables que se comparan, sino que también depende de la desviación y del número de sujetos de lá muestra, que en este caso es muy alto y actúa amplificatndo las pequeñas diferencias entre la medias. Teniendo presentes estas consideraciones, se analizan la significación de las diferencias entre todas las variables del cuestionario PAC, con especial atención, obviamente, a las que definen las dimensiones de la taxonomía de actitudes relacionadas con la ciencia utilizadas en este estudio.

La variable Actitud total tiene diferencias muy significa. tivas con todas las demás variables de actitudes relacionadas con la ciencia $(p<, 000)$. Respecto a las variables secundarias del cuestionario, las diferencias también son muy significativas en todos los casos, teniendo la significación más pequeña con la variable Salud $(t=$ $2,87 ; \mathrm{p}=, 004)$.

La variable Imagen de la ciencia tiene diferencias muy significativas con todas las demás variables de actitudes relacionadas con la ciencia $(p<, 001)$, aunque con relación a Curiosidad es más baja $(\mathrm{t}=3,37 ; \mathrm{p}=, 001)$. Respecto a las variables secundarias del cuestionario,

ACTITUDES RELACIONADAS CON LA CIENCIA

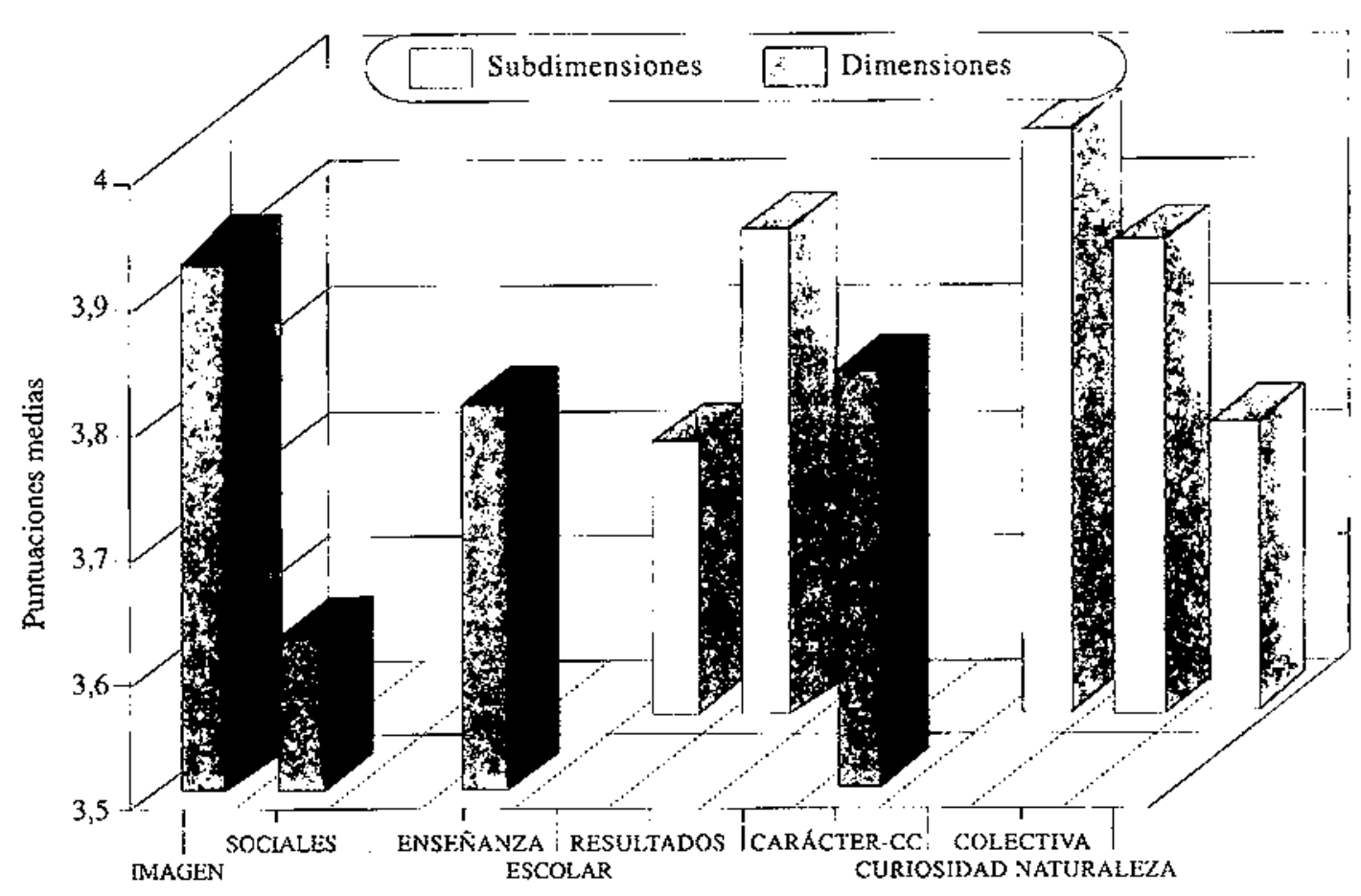

DIMENSIONES Y SUBDIMENSIONES TAXONOMIA 
las diferencias también son significativas en todos los casos, siendo las menos significativas las correspondientes a la variable Aburrida $(\mathrm{t}=2,98 ; \mathrm{p}=, 003)$.

La variable Sociales tiene la puntuación media más baja, y sus diferencias son muy significativas con todas las demás variables de actitudes y secundarias $(\mathrm{p}<, 000)$.

La variable Enseñanza tiene diferencias muy significativas con sus dos componentes (Escolar y Resultados) y con todas las demás actitudes, excepto con Características de la ciencia, que son bajas $(\mathrm{t}=-2,48 ; \mathrm{p}=013)$, aunque con las tres componentes de ésta también son muy significativas ( $\mathrm{p}<, 000$ ). La variable Escolar, componente de actitudes hacia la enseñanza de la ciencia, tiene diferencias muy significativas con todas las variables de actitudes excepto con Naturaleza de la ciencia, de la que no se diferencia significativamente. Con las variables secundarias del cuestionario PAC, las diferencias no son significativas con Mundo mejor y Salud. La otra componente de Enseñanza, denominada Resultados de la escuela, tiene diferencias muy significativas con todas excepto con la actitud hacia la ciencia como empresa Colectiva, y con la variable secundaria Aburrida.

La variable Caracteristicas de la ciencia tiene diferencias significativas con todas las demás variables de actitudes, incluso con sus tres componentes (Valores, Colectiva y Naturaleza de la ciencia), siendo bajas con Enseñanza. Con las demás variables secundarias, las diferencias también son muy significativas. La componente de actitudes relacionada con Valores de la ciencia. tiene diferencias muy significativas con todas las variables; Colectiva también, excepto con Aburrida ( $t=$ $-1,30 ; \mathrm{p}=, 193)$. Naturaleza no difiere significativamente de Mundo mejor y Salud. Existen diferencias significativas entre todas las variables de temas secundarios del cuestionario PAC, excepto en el caso de la variable Mundo mejor con Salud.

En resumen, el análisis de las diferencias entre las puntuaciones medias de las variables de actitudes y temas secundarios del cuestionario $P A C$ revela que estas diferencias son muy significativas en la mayorfa de los casos, de manera que, a pesar del pequeño valor numérico aparente de las diferencias entre los valores medios, debido al pequeño rango elegido al operacionalizar las variables de actitudes, las diferencias en actitudes de Ia amplia muestra seleccionada son muy diferentes según el objeto de la actitud relacionada con la ciencia que se trate. Por tanto, las preferencias actitudinales de Ios estudiantes hacia los distintos objetos de actitud de la taxonomía, operacionalizados en el PAC, tienen diferencias que marcan una gradación significativa entre ellas con el orden citado.

En la tabla II también figuran los coeficientes de fiabilidad de la escala total (en la variable Actitud) y cada una de las subescalas consideradas. La fiabilidad está medida en todos los casos como un coeficiente de consistencia interna, operacionalizado en este caso mediante eI parámetro alfa de Cronbach. El valor obtenido para la escala total es muy bueno, y se sitúa en la parte alta de la banda de los valores obtenidos para los estudios previos que aplicaron la escala original (WASP), por lo que nuestra versión (PAC) puede considerarse un instrumento muy satisfactorio para evaluar las actitudes relacionadas con la ciencia.

A la hora de evaluar la fiabilidad de las distintas subescalas debe tenerse en cuenta que una de las principales variables que afectan a la fiabilidad de una escala es la longitud (el número de f́tems) que forman el instrumento (igual que en todos los demás factores, las escalas más largas tienen mayor fiabilidad); por tanto, los valores directos que se muestran en la columna Alfa de la tabla no pueden utilizarse para discriminar la mayor o menor fiabilidad de las distintas subescalas, ya que tienen distintas longitudes. Para evitar este inconveniente, se ha empleado la fórmula de Spearman-Brown, que permite averiguar la fiabilidad para una longitud dada; mediante su aplicación, se han calculado todos los valores de la fiabilidad de todas las subescalas como si tuvieran una longitud de 50 ítems (como la escala totaI) y se han colocado en la columna Alfa corregida. Los valores corregidos obtenidos para las distintas subescalas son todavía mejores que los de la escala total, lo cual no es nada sorprendente, sino completamente lógico, ya que otro de los factores de los que depende la fiabilidad es la homogeneidad de los ítems que forman cada escala. Lógicamente, al agrupar los ítems en una subescala mediante algún criterio de afinidad se está haciendo más homogénea cada una de las subescalas, por 10 que la fiabilidad de estas (Alfa corregida) debería mejorar, como así se observa, siendo, en este caso, excelentes.

Puesto que estos valores corregidos están ya referidos todos a la misma longitud (en este caso la misma que la escala total, 50 ftems), es posible compararlas directamente entre sí. Dentro de los excelentes valores de fiabilidad obtenidos, las diferencias de fiabilidad entre las subescalas son tan mínimas que no permiten identificar diferencias importantes de fiabilidad entre las subescalas. No obstante, se observa que la subescala con mejor fiabilidad y la que tiene la fiabilidad más baja son justamente las dos subescalas de Enseñanza, esto es, Ciencia escolar y Resultados de la enseñanza, respectivamente.

\section{DISCUSIÓN Y CONCLUSIONES}

Este estudio se propone dos objetivos principales: por un lado, ofrecer una evaluación diagnóstica de las actitudes relacionadas con la ciencia con el instrumento de medida aplicado y, por otro, ejemplificar la operacionalización, sobre el cuestionario utilizado, de la taxonomía de las actitudes relacionadas con la ciencia propuesta como instrumento teórico y conceptual, para analizar su capacidad de superar algunos de los defectos metodológicos endémicos que afectan a la investigación de las actitudes. 
La operacionalización de las actitudes relacionadas con la ciencia sobre el instrumento estandarizado empleado muestra la flexibilidad y capacidad de la taxonomía para identificar las distintas dimensiones de actitudes relacionadas con la ciencia sobre un conjunto de ítems que no han sido diseñados expresamente para este objetivo. En relación con este punto, se puede considerar que el cuestionario ha permitido ejemplificar las distintas dimensiones de la taxonomía con suficiente validez y fiabilidad, y analizar con más detalle, en base a esta categorización, los resultados diagnósticos obtenidos con cl cuestionario.

No obstante, cabc observar que la representatividad del cuestionario es particularmente escueta en dos de las dimensiones de la taxonomf́a: los valores científicos y Ios temas sociales en la ciencia. Respecto a los valores que asume el trabajo científico, el instrumento sólo explicita la curiosidad, dejando de lado otros valores importantes de la ciencia y los científicos tales como la búsqueda de la objetividad, la honestidad, las motivaciones, el compromiso social, etc. Respecto a los temas sociales de las ciencia, se podría extender el número de temas con incidencia social incluidos en el cuestionario a temas como los que se refieren a la conservación del medio ambiente (efecto invernadero, agujero de la capa de ozono, lluvia ácida, vertidos contaminantes, etc.), los cuales muestran la ambivalencia de la ciencia (tecnologías de guerra, armamentos, energía nuclear, etc.) o las relaciones de la ciencia y la sociedad (control de la ciencia, información al público de los descubrimientos científicos, contríbución de la ciencia a solucionar o agravar los problemas sociales, etc.). En ambos aspectos, la inclusión de ítems que recojan estos temas podría contribuir a hacer más completo y consistente el cuestionario de evaluación de actitudes relacionadas con la ciencia.

Desde la perspectiva metodológica, el cuestionario PAC aplicado está construido con cuestiones tipo Likert, un formato que no ha sido muy utilizado en investigaciones anteriores en nuestro país, a pesar de que es uno de los más universales en la literatura internacional, $y$, por tanto, puede servir como pauta para futuros desarrollos y estudios. La fiabilidad obtenida para el protocolo global tiene un valor muy bueno y resulta todavía mejor para las distintas subescalas definidas en él, que se corresponden con las dimensiones de la taxonomía de actitudes relacionadas con la ciencia, lo cual avala también la adecuación de las dimensiones definidas, e indirectamente constituye, también, un respaldo empírico a la validez y utilidad de la taxonomía.

Desde la perspectiva del diagnóstico de las actitudes relacionadas con la ciencia evidenciadas en la muestra de estudiantes, el principal resultado es la identificación de una buena actitud general media hacia la ciencia, obtenida en la muestra completa de estudiantes. La actitud media hacia la ciencia se sitúa en valores moderadamente positivos, aunque existen diferencias entre las distintas dimensiones de la taxonomía de las actitudes. La mejor puntuación es obtenida por la imagen de la ciencia, y la peor puntuación se obtiene para los aspectos sociales de la ciencia, siendo intermedias las actitudes relacionadas con tos aspectos escolares y las características del conocimiento científico. Entre las distintas subdimensiones definidas para las dimensiones de ensenanza y características de la ciencia, las actitudes más bajas obtenidas se refieren a la enseñanza-aprendizaje en la escuela y a los aspectos relacionados con la naturaleza de la ciencia, pero sin llegar, en ninguno de los dos casos, al mínimo de puntuación actitudinal marcada por la puntuación de los aspectos sociales. Las diferencias de actitud entre diferentes grupos de estudiantes tales como hombres y mujeres, cientificos y no científicos, jóvenes y menos jóvenes se ha estudiado en otro lugar (Vázquez y Manassero, 1996a).

Las diferencias significativas obtenidas entre las puntuaciones de las distintas dimensiones y subdimensiones constituyen un nuevo aval en favor de la utilidad y adecuación de la taxonomía de actitudes relacionadas con la ciencia. En efecto, las distintas dimensiones de la taxonomía corresponden a diferentes objetos de actitud conceptuales; las diferencias significativas obtenidas entre estas dimensiones suponen una confirmación cmpírica de la validez de la distinción teórica marcada por la taxonomía. En suma, los distintos objetos de actitud relacionados con la ciencia definidos por la taxonomía resultan ser empíricamente productivos, ya que permiten discriminar aspectos actitudinales en el área de las actitudes relacion ddas con la ciencia, que resultan ser realmente diferentes.

Los resultados que muestran una buena actitud de los estudiantes hacia la ciencia no son muy habituales (Espinosa y Román, 1991; Ortega et al., 1992), especialmente en lo que se refiere a estudios realizados en otros países (James y Smith, 1985; Simpson y Oliver, 1985, 1990), por lo que este resultado obtenido aquí con estudiantes españoles es un contrapunto a la tradicional actitud desfavorable hacia la ciencia de los estudiantes. Sin embargo, la problemática, ya citada, de falta de homologación y validez de los instrumentos de medida de las actitudes, así como las deficiencias y diferencias en la definición del objeto de actitud son obstáculos que no permiten llevar más adelante una comparación fiable, por lo que debemos limitarnos a dejar constancia de esta inflexión: los estudiantes de nuestra muestra no exhiben una actitud negativa en relación con la ciencia, sino moderadamente positiva, al menos en los aspectos actitudinales diagnosticados con el cuestionario aplicado.

Las consecuencias de cste estudio para la práctica de la enseñanza de las ciencias están condicionadas porque los objetivos principales de este estudio son de diagnóstico de las actitudes relacionadas con la ciencia y de validación de la taxonomía, de modo que, aunque el tema de las actitudes tiene un flanco didáctico de indudable interés (el diseño de contenidos y metodología para la educación de las actitudes), los comentarios y sugerencias que se proponen estarán referidos, exclusivamente, a los objetivos señalados.

Así, una de las principales consecuencias que se desprenden de la tevisión realizada es la necesidad de 
definir con precisión el objeto de actitud, para lo cual la taxonomía aporta una serie de categorías conceptuales, fundamentadas teóricamente, para facilitar esta precisión. En un nivel puramente reactivo, la precisión requiere evitar algunos de los defectos más comunes cometidos en estudios investigaciones de actitudes realizados en el pasado, mientras que, desde una perspectiva proactiva, la definición requiere especificar con la mayor exactitud posible el objeto de actitud relacionada con la ciencia que se trate.

En un nivel reactivo existe una creencia ingenua, muy extendida, sobre las actitudes en ciencias, que consiste en identificar actitud hacia la ciencia con interés del estudiante por aprender ciencia. No cabe calificar de errónea esta creencia ingenua, porque simplemente se trata de una conceptualización de mero sentido común, sin mayor fundamentación teórica, pero que tiene una influencia negativa sobre la conceptualización y definición de las actitudes, derivada del carácter único, excluyente, implícito y limitado con que los profesores asumen esta concepción. Así, muchos profesores sóloentienden la actitud en el sentido único de interés del estudiante por aprender y estudiar, de modo que no conciben otro concepto de actitud distinto, por lo que esta creencia ingenua resulta excluyente, ya que no deja lugar para otros posibles conceptos de actitudes distintos, que puedan competir con éste. El carácter único condiciona también que esta creencia se mantenga implícita, es decir, no manifiesta, puesto que al suponerse única no se necesita la expresión explícita de la misma, pues se considera, erróneamente, que es asumida igualmente por todos, y por esa misma razón constituye una concepción limitada, o sea, con sus propios horizontes cerrados sobre sí misma. En suma, esta identificación ingenua de actitud hacia la ciencia con interés hacia el aprendizaje de la ciencia constituye un reduccionismo conceptual y metodológico, que limita los horizontes actitudinales, excluyendo otras posibles conceptualizaciones de actitudes diferentes. La taxonomía de actitudes relacionadas con la ciencia pretende superar esta visión ingenua de las actitudes, subrayando el carácter plural de éstas actitudes, es decir múltiples y muy interesantes objetos de actitud en ciencia; y, en consecuencia, es necesario defínir con precisión cada objeto de actitud que se considere. A su vez, las dimensiones que forman la taxonomía, una de las cuales incluiría la que se ha denominado creencia ingenua sobre las actitudes (actitud hacia Ios elementos escolares de la ciencia), constituyen un inventario genérico de actitudes que puede ser una guía fecunda, no sólo para la definición precisa de actitudes, sino también para un diseño curricular de contenidos actitudinales en la enseñanza de las ciencias (para una mayor justificación ver Vázquez y Manassero, 1995).

La evaluación de las actitudes, en general, y de las actitudes relacionadas con la ciencia, en particular, es otro de los obstáculos en contra de una educación de las actitudes, debido a múltiples razones que hemos examinado en más profundidad en otro artículo (Vázquez y Manassero, 1996b), pero que se pueden sintetizar en la afirmación de que las actitudes no pueden (o deben) evaluarse. El carácter diagnóstico y evaluativo de actitudes que tiene este estudio constituye un argumento y un ejemplo en favor de la tesis de la evaluabilidad de las actitudes; este estudio muestra una forma sencilla y simple de evaluar las distintas dimensiones de la taxonomía de las actitudes relacionadas con la ciencia, mediante una prueba de papel y lápiz, que, aunque no es la única forma posible de evaluar actitudes, es la más semejante a los exámenes y pruebas corrientemente utilizados por los profesores para evaluar conocimientos y procedimientos. Los múltiples cuestionarios citados en la introducción y otros más recientes, sobre los que se trabaja en la actualidad, son intrumentos a disposición đel profesorado para realizar la evaluación de actítudes relacionadas con la ciencia con diversas metodologías y para superar la prevención que suscita el tema de la evaluación de las actitudes (Aikenhead, Fleming y Ryan, 1987; Aikenhead y Ryan, 1992; Rubba y Harkness, 1993).

En suma, este estudio valida y ejemplifica la utilización empírica de la taxonomía de actitudes relacionadas con la ciencia como guía para la evaluación de las diferentes dimensiones de la misma, al mismo tiempo que sus resultados evidencian las diferencias entre los distintos objetos actitudinales de la taxonomía. Desde una perspectiva diagnóstica, se demuestra la posibilidad de evaIuar las actitudes hacia la ciencia mediante una sencilla prueba de papel y lápiz y se respalđa la capacidad potencial de la taxonomía para contribuir a la superación del reduccionismo de creer en una única versión ingenua de actitud hacia la ciencia, facilitar una definición más precisa de los distintos objetos actitudinales y también afrontar un diseño de los contenidos actitudinales para una educación integral de las actitudes relacionadas con la ciencia.

\section{NOTA}

Estudio financiado por el Ministerio de Educación y Ciencia a través del programa de Ayıdas a la Investigación Educativa de 1992 


\section{REFERENCIAS BIBLIOGRÁFICAS}

ACEVEDO, J.A. (1993a). ¿Qué piensan los estudiantes sobre la clencia? Ln enfoque CTS. Enseñanza de las Ciencias, Vol. extra, IV Congreso Intemacional sobre investigación en la didáctica de las ciencias y las matemáticas, pp. 11-12, Barcelona, 13-16 de septiembre.

\CEVEDO, J.A. (1993b). Actitudes hacia el aprendizaje de las ciencias físicas, naturales y matemáticas en el BUP y COU. Un estudio sobre tres dimensiones. Enserianzade las Ciencias, Vol. extra, IV Congreso Internacional sobre investigación en la didáctica de las ciencias y las matemáticas, pp. 13-14, Barcelona, 13-16 de septiembre.

AIKENHEAD, G.S., FI.FMING, R.G. y RYAN, A.G. (1987). High School Graduates' Beliefs About Science-TechnologySoctety. I. Methods and Issues in Monitoring Students Views. Science Education, 71(2), pp. 145-161.

AIKENHEAD, G.S. y RYAN, A.G. (1992). The development of a new instrument: "Views on Science-Technology-Society» (VOSTS). Science Education, 76(5), pp. 477-492.

AI.I.EN, H., Jr. (1959). Atitudes of certain high school sentor toward science and scientific careers. Nueva York: Martin Press, Inc.

ÁVAREZ, M., SONEIRA, G. y PIZARRO, I. (1993). Como percibe el alumnado algunas interacciones entre CienciaTecnologia-Género-Sociedad. Inseñanza de las Ciencias, Vol. extra, IV Congreso Internacional sobre investigación en la didactica de las ciencias y las matemáticas, pp. 19-20, Barcelona, 13-16 de septiembre.

ARANA, J., ESCLDFRO, T., GARCFS, R. y PAI.ACIAN, C. (1985). Imagen de las asignaturas de ciencias en la transición de la educación básica a la secundaria. Enseñanza de las Ciencias, Vol. extra, I Congreso Internacional sobre investigación en la didáctica de las ciencias y las matemáticas, p. 20, Barcelona.

BII.I.FH, V.Y. y 7.AKHIARIADES, G.A. (1975). The development and application of a scale for measuring scientific attitudes. Science Education, 59, pp. 155-165.

BRA'T', M. (1984). Iuther Comments on the Validity Studies of Attitude Measures in Science Education. Journal of Research in Science Teaching, 21, pp. 951.

ESCUDERO, T, y LACASTA, E. (1984). Las actitudes científicas de los futuros maestros en relación con sus conocimientos. Enseñanza de las Ciencias, 2, pp. 175-180.

ESCUDERO ESCORZA, T. (1985). Las actitudes en la enseñanza de las ciencias: un panorama complejo. Revistade Educación, 278 , pp. 5-25.

ESPINOSA, J. y ROMÁN, T. (1991). Actitudes hacia la ciencia $y$ asignaturas pendientes: dos factores que afectan al rendimiento en ciencias. Enseñanza de las Ciencias, 9, pp. 151-154.

GARDNER, P.L. (1975). Attitudes to science: A review. Studies in Science Education, 2, pp. 1-41.

GAUIID, C.F. y HUKINS, A.A. (1980). Scientific attitudes: A review. Studies in Science Education, 7, pp. 129-161

GIL, D., MAR'ÍNE\%, J. y SENENT, F. (1987). Actitudes y expectativas del protesorado ante la resolución de problemas de física. Enseñanza de las Ciencias, Vol. extra, II Congreso
Internacional sobre investigación en la didactica de las ciencias y las matemáticas, pp. 203-205, Valencia, 23-25 de septiembre.

GÓMEZ, C. y CERVERA, S. (1989). Actitudes y cambio conceptual en la educación ambiental. Enseñanza de las Ciencias, Vol. extra, II Congreso Congreso Intemacional sobre investigación en la didkictica de las ciencias y las matemáticas, pp. 209-211, Santiago de Compostela, 20-22 de septiembre.

GUASCH, E, DE MANUEI, J., y GRAU, R. (1993). La imagen de la ciencia en alumnos y profesores. I a influencia de los medios de comunicación. Ensentanza de las Ciencias. Vol. extra, IV Congreso Internacional sobre investigacion en la didáctica de las ciencias y las matemáticas, pp. 77-78. Barcelona, 13-16 de septiembre.

JAMES, R.K. y SMITH, S. (1985). Alienation of Students from Science in Grades 4-12. Science Edtcation, 69, pp. 39-45.

KLOPFER, L.E. y COOLEY, W.W. (1961) Testof understandins science: form $W$. Princeton, $N$ J L Lucational Testing Service.

KO7I.OW, M.J. y NAY, M.A. (1976). An approach to measuring scientific attitudes. Science Education, 60, pp. 147-172.

IOWERY, L.F. (1966). Development of an attitude measurement instrument for science education. School, Science and Muth, 66, pp. 494-502.

MORENO, M.D. y GIL, D. (1987). La medida de los estudiantes de BLP hacia la Física. Enseñanza de las Ciencias, Vol. extra, II Congreso Internacional sobre investigación en la didactica de las ciencias y las matemáticas, pp. 268-270. Valencia, 23-25 de septiembre.

MUNBY, H. (1983). Thirty studies involving the "Scientific Attitude Inventorys: What confidence can we have in this instrument? Journal of Research in Science Teaching, 20. pp. 141-162.

MURPHY, G, (1968). Content versus process centered biology laboratories, part II: The development of knowledge, scientific attitudes, problem-solving ability, and interest in biology. Science Education, 52, pp. 148-162.

ORTEGA, P., SAURA, J.P., MINGUEZ, R., GARCÍA, A. y MARTINEZ, D. (1992). Diseño y aplicación de una escali de actitudes hacia el estudio de las ciencias experimentales. Enseñanza de las Ciencias, 10, pp. 295-303.

RUBBA, P.A. y HARKNESS, W.L. (1993). Examination of preservice and in-service secondary science teachers' beliefs about Science-Technology-Society interactions. Science Education, 77, pp. 407-431.

SCHIBECI, R.A. (1984). Attitudes to science: Un update. Studies in Science Education, 11, pp. 26-59.

SCHIWIRIAN, P.M. (1968). On measuring attitudes toward science. Science Education, 52, pp. 172-179.

SERRANO, T. (1986). La imagen de los científicos en los alumnos al finalizar el ciclo medio. Documentos IEPS, Monografias, 1. Madrid: IEPS.

SERRANO, T. (1988). Actitudes de los alumnos y aprendizaje de las ciencias. Un estudio longitudinal. Investigación en la Escuela, 5, pp. 29-38. 
SHRIGLEY, R.L. y KOBALLA, T.R. (1984). Attitude measurement: judging the emotional intensity of Likerttype science attitude statements. Joumal of Research in Science Teaching, 21, pp. 111-118.

SHRIGLEY, R.L. y KOBALLA Jr., T.R. (1992). A decade of attitude research based on Hovland's leaming model. Science Education, 76, pp. 17-42.

SIMPSON, R.D. y OLIVER, I.E. (1985). Attitude Toward Science and Achievement Motivation Profiles of Male and Female Science Students in Grades Six through Ten. Science Education, 69(4), 511-526.

SIMPSON, R.D. y OLIVER, J.E. (1990). A Summary of Major Influences on Attitude Toward and Achievement in Science Among Adolescent Students. Science Education, 74(1), pp. $1-18$.

SOLBES, \. y VILCHES, A. (1989). Interacciones CienciaTécnica-Sociedad: un instrumento de cambio actitudinal. Enseñanza de las Ciencias, 7, pp. 14-20.

SOLBES, I. y VILCHES, A (1992). El modelo constructivista y las relaciones Ciencia-Técnica-Sociedad (CTS). Enseñanza de las Ciencias, 10, pp. 181-186.

SONEIRA, G., LIRES, M.A. y PIZARRO, I. (1993). Actitudes del atumnado ante las materias optativas de ciencias y tetras. Diferencias de género. Enseñanza de las Ciencias, Vol. extra, IV Congreso Internacional sobre investigación en la didáctica de las ciencias y las matemáticas, pp. 137-138, Barcelona, 13-16 de septiembre.

VÁZQUEZ, A. y MANASSERO, M.A. (1995). Actitudes relacionadas con la ciencia: una revisión conceptual. Enseñanza de las Ciencias, 13(3), pp. 337-346.

VÁZQUEZ, A. y MANASSERO, M.A. (1996a). Factores determinantes de las actitudes relacionadas con la ciencia. Revista Española de Pedagogía, 203, pp. 43-77.

VÁZQUEZ,A. y MANASSERO, M.A. (1996b). El aprendizaje significativo en ciencias: integración del cambio conceptual, metodológico y actitudinal. (Manuscrito enviado para publicación.)

WAREING, C. (1982). Developing the WASP: Wareing's attitudes toward science protocol. Journal of Research in Science Teaching, 19, pp. 639-645.

WAREING, C. (1990). A Survey of Antecedents of Attitudes Toward Science. Journal of Research in Science Teaching, 27 , pp. $371-386$.

WEINHOLD, J.D. (1970). An attempt to measure the scientific attitudes of elementary school teachers. Dissertation Abstracts International, 31, 1647-A.

ZEIDLER, D.I. (1984). Thirty studies involving the «Scientific Attitude Inventory": what confidence can we have in this instrument. Journal of Research in Science Teaching, 21, pp. $341-342$.

[Artículo recibido en octubre de 1995 y aceptado en noviembre de 1996.] 


\section{APANDICE \\ PROTOCOLO DE ACTYTUDES RFI,ACIONADAS CON I.A CIENCIA (PAC) \\ INSTRUCCIONES}

Este instrumento está diseñado pora valorar tus actitudes hacia la ciencia. No existen respuestas correctas o incorrectas sino que sólo se desca conocer tu opinión sincera sobre cada frase. Por favor. lee atentamente cada frase y señala con un círculo la letra que corresponde a tus propios sentimientos sobre la frase, valorada con las siguientes categorias:

$$
\begin{aligned}
& A T=\text { Acuerdo total } \\
& A=\text { Acuerdo } \\
& I=\text { Indecisión } \\
& D=\text { Desacuerdo } \\
& D T=\text { Desacuerdo total }
\end{aligned}
$$

- Completa los datos personales siguientes. Escribe sobre los puntos o subraya la respuesta correcta.

EDAD SI:XO: Hombre / Mujer

- Responde rodeando con un círculo la opción elegida para cada cuestión.

1. Tenemos un mundo mejor para vivir gracias a la ciencia.

2. A nadie le gusta la ciencia.

3. La ciencia ayuda a ahorrar tiempo y esfuerzo

4. La ciencia es muy difícil de aprender.

5. Las enfermedades pueden curarse gracias a la ciencia.

6. Cuanto mayor conocimiento cientiftco existe, más preocupaciones hay para nuestro mundo.

7. La ciencia no es aburrida.

8. La ciencia ayuda a la gente en todos los sitios.

9. La ciencia es sensata.

10. Me siento mal ślo de pensar en la ciencia.

11. La curiosidad es lo esencial de la ciencia.

12. La gentc vive más saludablemente gracias a la ciencia.

13. La ciencia no puede tesolver los problemas energéticos.

14. Para destacar en ciencia hay que ser muy inteligente.

15. Los alumnos estudian ciencia porque se les obliga.

16. La ciencia es el camino para conocer el mundo en que vivimos.

17. La ciencia estimula la curiosidad.

18. No hay nacla mejor que trabajar en ciencia.

19. La ciencia es algo realmente valioso.

20. Conocer la I.una y los planetas a través de la ciencia nos ayuda aquí en la Tjerta.

21. En las clases de ciencia no hay actividad.

22. La pcor materia escolar es la ciencia.

23. I.a ciencia debería ser eliminada de las escuelas.

24. La gente tienc una vida más larga gracias a la ciencia.

25. Los alumnos en clase de ciencia son como robots.

26. La ciencia desanima la curiosidad.

27. La cjencia nos ayuda a pensar mejor.

28. Estudiar ciencia es una pesadilla.

29. I a gente scria mejor estudiante si no tuviera ciencia.

30. La ciencia no tiene mucho sentido para gente que no son científicos.

31. La ciencia nos ayuda a evitar catastofres.

32. Un futuro mejor depende de la ciencia.

33. La cicncia nos enseña a prepararnos para el futuro.

34. La ciencia es un riesgo para la salud.

35. L.a vida sería monótona sin ciencia.

36. No deberíamos haber enviado gente a la Luna.
AT A I D DT

AT $\wedge$ I D DT

AT $A$ I D DT

$A T$ A I D DT

AT AID DI

$A T A I D$ DT

AT $\wedge$ I D DT

AT $\wedge$ I D DT

AT $\wedge$ I D DT

AT A I D DT

AT A I D DI

AT A I D DT

AT A I D DI

AT A I D DT

AT A I D DT

$\wedge T \wedge$ I D DT

AT A I D DT

AT A I D DT

AT A I D DT

AT A I D DT

AT A I D DT

$A T A I D D T$

AT A I D DT

AT A I D DT

NT A I D DT

AT A I D IDT

AT A I D DT

AT A I D DT

AT A I D DT

AT A I D DT

AT A I D DT

AT A I D DT

AT A : D D'

AT A I D DT

$A T$ A I D DT

AT A I D DT 
37. La ciencia es completamente aburida.

AT A I D DT

38. La ciencia es una excusa para juguetear.

AT A I D DT

39. La ciencia es detestable.

40. La ciencia es extremadamente útil.

41. La ciencia es necesaria para todos.

42. Estudiando ciencia se satisface la curiosidad.

43. La ciencia no tiene utilidad.

44. La ciencia nos enseña a aceptar muchas opiniones diferentes.

AT A I D DT

AT A I D DT

AT A I D DT

AT A I D DT

45. La ciencia nos defiende de la superstición.

AT A I D DT

46. Apreciar las ideas nuevas es valioso en ciencia.

47. Una vez aceptado, el conocimiento científico no puede ser cambiado.

48. La ciencia es una superstición.

49. La ciencia parece ser muy interesante.

50. Estudiar ciencia sirve a la gente, incluso cuando deja la escuela.

AT A I D DT

AT A I D DT

AT A I D DT

AT A I D DT

AT A I D DT

AT A I D DT

AT A I D DT 
